\title{
ORTHOGONAL REPRESENTATIONS OF ALGEBRAIC GROUPS
}

\author{
BY \\ FRANK GROSSHANS
}

Introduction. Let $G_{1}$ and $G$ be connected semisimple algebraic groups defined over a field $K$ of characteristic zero and assume that there is an isomorphism $f$ of $G_{1}$ onto $G$ which is defined over $\bar{K}$, the algebraic closure of $K$. If $\rho: G \rightarrow G L(V)$ is an absolutely irreducible (finite-dimensional) representation of $G$ defined over $K$, then $\rho \circ f$ is an absolutely irreducible representation of $G_{1}$ defined over $\bar{K}$. Satake [7, p. 230] has shown that there is a field $K_{1}$ which is a finite extension of $K$, a (unique) central simple division algebra $K \#$ defined over $K_{1}$, a finite-dimensional right vector space $V_{1}$ over $K \#$, and a $K_{1}$-homomorphism $\rho_{1}: G_{1} \rightarrow G L\left(V_{1} / K \#\right)$ (the group of all nonsingular $K \#$-linear endomcrphisms of $\left.V_{1}\right)$ such that $(\rho \circ f)(g)$ $=\theta_{1}\left(\rho_{1}(g)\right)$ for all $g \in G_{1}$ where $\theta_{1}$ is a unique absolutely irreducible representation of End $\left(V_{1} / K \#\right)$ (the algebra of all $K \#$-linear endomorphisms of $\left.V_{1}\right)$ onto End $(V)$.

In this paper we are interested in the case where $K=K_{1}$ and where there are invariant forms on $V$ and $V_{1}$. More precisely, we state the following two problems.

Problem 1. Assume that $K \#=K$ and that there are invariant bilinear forms $B$ on $V$ and $B_{1}$ on $V_{1}$ which are defined over $K$. What is the relationship between these two forms over $K$ ? Of course, if $B$ is alternating, so is $B_{1}$ and both are determined by $\operatorname{dim} V=\operatorname{dim} V_{1}$. Hence, we shall always take $B$ and $B_{1}$ to be symmetric.

Problem 2. Assume that $K \#$ is a nontrivial division algebra over $K$ (i.e., $K \# \neq K)$ and that there is an invariant bilinear form $B$ on $V$ and an invariant $\varepsilon$ hermitian form $F(\varepsilon=+1$ or -1$)$ on $V_{1}$ both of which are defined over $K$. What is the relationship between these two forms over $K$ ?

We are especially interested in the case $K=Q_{\mathfrak{k}}$, a $\mathfrak{p}$-adic field. (In a future paper, we shall discuss the case $K=\boldsymbol{R}$.) Here, some simplifications are immediately available. In Problem 2, it can be shown [7, p. 232] that $K \#$ has an involution of the first kind; but over $Q_{\mathfrak{p}}$, it is known that the only such division algebra is the quaternion division algebra. Furthermore, it is known that a hermitian form on a finite-dimensional vector space over a quaternion division algebra defined over $Q_{\mathfrak{p}}$ is determined only by the dimension of the vector space. Therefore, in Problem 2 we shall always take $F$ to be skew-hermitian; in the case where $K \#$ is a quaterion division algebra, this means that the form $B$ is symmetric [7, p. 233].

If $W$ is a vector space defined over $K$ and if $S$ is a symmetric form on $W$ which is also defined over $K$, then three invariants can be associated with the pair $(W, S)$,

Received by the editors February 20, 1968. 
namely, (1) the dimension of $W, \operatorname{dim} W,(2)$ the discriminant of $S, \Delta(S)$, and (3) the Hasse invariant, $c(S)$. In answering Problem 1, we describe these three invariants of $B_{1}$ in terms of those of $B$. Over $Q_{\mathfrak{p}}$, these invariants completely describe a symmetric form.

Similarly, in Problem 2 we deal with two invariants of the space $\left(V_{1}, F\right)$, namely, (1) the dimension of $V_{1}$ (over $K \#$ ), $\operatorname{dim} V_{1}$, and (2) the discriminant of $F, \delta(F)$. We describe these invariants in terms of the invariants of $B$. Over $Q_{\mathfrak{p}}$, the two invariants above completely describe a skew-hermitian form.

The answers to the questions above fall into two main parts. In Part I, we assume that the isomorphism $f: G_{1} \rightarrow G$ is of inner type, i.e., for each $\sigma \in \Gamma$ (the Galois group of $\bar{K}$ over $K), f^{-\sigma} \circ f=I_{g_{\sigma}}$ where $g_{\sigma} \in G_{1}$ and $I_{g_{\sigma}}(g)=g_{\sigma} g g_{\sigma}^{-1}$ for all $g \in G_{1}$. (By $f^{-\sigma}$, we shall always mean $\left(f^{-1}\right)^{\sigma}$.)

For absolutely simple groups $G_{1}$, it is well known that there is a Chevalley group $G$ defined over $K$ and an isomorphism $f: G_{1} \rightarrow G$ defined over $\bar{K}$ of inner type, except possibly when $G_{1}$ is of type $A_{n}, D_{n}$, or $E_{6}$. These last three cases are discussed in Part II.

This paper is a portion of the author's doctoral thesis written at the University of Chicago. He is very grateful to Professor Ichiro Satake who was his advisor and to the National Science Foundation for supporting his graduate study.

\section{PART I}

1.1. The standard situation. Throughout this part, we shall assume that $f$ is of inner type, i.e. $f^{-\sigma} \circ f=I_{g_{\sigma}}$ for each $\sigma \in \Gamma$. The elements $g_{\sigma}$ in $G_{1}$ are determined modulo the center of $G_{1}, Z\left(G_{1}\right)$, and so for $\sigma, \tau \in \Gamma$, the element $c_{\sigma, \tau}=g_{\sigma}^{\tau} g_{\tau} g_{\sigma \tau}^{-1}$ are in $Z\left(G_{1}\right)$. It follows that the cohomology class $\left(c_{\sigma, \tau}\right)$ of the 2-cocycle $c_{\sigma, \tau}$ of $\Gamma$ in $Z\left(G_{1}\right)$ is independent of the choice of elements $g_{\sigma}$. This 2-cocycle will play an important role in what follows.

Let $\rho: G \rightarrow S O(V, B)$ be an absolutely irreducible orthogonal representation defined over $K$ and assume that $B$ is also defined over $K$. In general, such a representation will be denoted by the triple $(V, \rho, B)$ and will be called an orthogonal representation of $G$ defined over $K$. Then $\rho \circ f$ is an orthogonal representation of $G_{1}$ defined over $\bar{K}$ and, setting $A_{\sigma}=(\rho \circ f)\left(g_{\sigma}^{-1}\right)$, we have that for each $\sigma \in \Gamma$

$$
(\rho \circ f)^{\sigma}(g)=A_{\sigma}(\rho \circ f)(g) A_{\sigma}^{-1}
$$

for all $g \in G_{1}$. Also, by definition of $A_{\sigma}$ and (1), it follows that

$$
A_{\sigma}^{\tau} A_{\tau}=(\rho \circ f)\left(c_{\sigma, \tau}^{-1}\right) A_{\sigma \tau}
$$

for all $\sigma, \tau \in \Gamma$. The continuous 2-cocycle $(\rho \circ f)\left(c_{\sigma, \tau}\right)$ defines $K \#$ as a normal division algebra if we require that $c(K \#) \sim\left((\rho \circ f)\left(c_{\sigma, \tau}\right)\right)[7$, p. 227].

1.2. Problem 1. Our concern in this section is the case where $\left((\rho \circ f)\left(c_{\sigma, \tau}\right)\right) \sim 1$. As we shall see, this is the case of Problem 1. However, before proving the theorem describing completely this situation, we need two lemmas. 
LEMMA I.1. Assume that $\left((\rho \circ f)\left(c_{\sigma, \tau}\right)\right) \sim 1$. Then there exist elements $h_{\sigma}$ in $G_{1}$ such that $h_{\sigma} \equiv g_{\sigma} \bmod Z\left(G_{1}\right)$ and $(\rho \circ f)\left(h_{\sigma, \tau}^{-1} h_{\sigma}^{\tau} h_{\tau}\right)=1$ for all $\sigma, \tau \in \Gamma$.

Proof. We set $d_{\sigma, \tau}=(\rho \circ f)\left(c_{\sigma, \tau}\right)$ for all $\sigma, \tau \in \Gamma$. Then, as is well known, since $d_{\sigma, \tau}$ is a 2-cocycle of $\Gamma$ in $\{+1,-1\}$ which is equivalent to 1 , there exist elements $a_{\sigma}$ in $\{+1,-1\}$ for each $\sigma \in \Gamma$ such that $d_{\sigma, \tau}=a_{\sigma}^{\tau} a_{\tau} a_{\sigma, \tau}^{-1}$.

If $\operatorname{dim} V \equiv 1(2)$, it is immediate that the elements $d_{\sigma, \tau}$ are always 1 as can be seen by taking determinants of both sides of (2). The case where $\operatorname{dim} V \equiv 0$ (2) is harder; however, if $d_{\sigma, \tau}$ is always 1 then there is nothing to prove. Therefore, we may assume that there is an element $z \in Z\left(G_{1}\right)$ such that $(\rho \circ f)(z)=-1$. In particular, for each $\sigma \in \Gamma$, there is an element $z_{\sigma} \in Z\left(G_{1}\right)$ such that $(\rho \circ f)\left(z_{\sigma}\right)=a_{\sigma}$. Using these $z_{\sigma}$, we define $h_{\sigma}$ to be $g_{\sigma} z_{\sigma}$. It is easy to see that these $h_{\sigma}$ satisfy the conditions above and so this lemma is proved.

From now on, we shall assume that the $g_{\sigma}$ are chosen so that $(\rho \circ f)\left(c_{\sigma, \tau}\right)=1$ for all $\sigma, \tau \in \Gamma$. Actually, in practice this choice is frequently trivial, for in many cases $(\rho \circ f)\left(Z\left(G_{1}\right)\right)=\{1\}$. Also, we shall assume that $G_{1}$ is simply connected. This assumption will be removed following the proof of Theorem I.1.

Denote the "spin group" of $B$ by Spin $(B)$ and let $\pi$ be the canonical mapping from Spin $(B)$ onto $S O(V, B)$. It is known that $\pi$ is defined over $K$ and that its kernel is $\{+1,-1\}$. Since $G_{1}$ is simply connected, there is a (polynomial) map $\rho_{s}: G_{1} \rightarrow \operatorname{Spin}(B)$ such that $\pi \circ \rho_{s}=\rho \circ f$. We define elements $\bar{A}_{\sigma} \in \operatorname{Spin}(B)$ by $\bar{A}_{\sigma}=\rho_{s}\left(g_{\sigma}^{-1}\right)$. Then $\pi\left(\bar{A}_{\sigma}\right)=A_{\sigma}$ and the system $\left\{\bar{A}_{\sigma}\right\}$ satisfies the relation $\bar{A}_{\sigma}^{\tau} \bar{A}_{\tau}$ $=e_{\sigma, \tau} \bar{A}_{\sigma \tau}$ where each $e_{\sigma, \tau}$ is +1 or -1 .

LEMMA I.2. Let $\rho_{s}: G_{1} \rightarrow \operatorname{Spin}(B)$ be such that $\pi \circ \rho_{s}=\rho \circ f$ and assume that each $(\rho \circ f)\left(c_{\sigma, \tau}\right)=1$. Then the $e_{\sigma, \tau}$ above are given as follows: $e_{\sigma, \tau}=\rho_{s}\left(c_{\sigma, \tau}\right)$.

Proof. For each $\sigma \in \Gamma$, we have $\pi \circ \rho_{s}^{\sigma}=(\rho \circ f)^{\sigma}=A_{\sigma}(\rho \circ f) A_{\sigma}^{-1}=\pi\left(\bar{A}_{\sigma} \rho_{s} \bar{A}_{\sigma}^{-1}\right)$. So $\rho_{s}^{\sigma}(g)=e(g) \bar{A}_{\sigma} \rho_{s}(g) \bar{A}_{\sigma}^{-1}$ where $e(g)=+1$ or -1 . But, since $G_{1}$ is connected, $e(g)$ is always 1 and so $\rho_{s}^{\sigma}(g)=\bar{A}_{\sigma} \rho_{s}(g) \bar{A}_{\sigma}^{-1}$ for all $g \in G_{1}$. Using this fact, the lemma follows immediately.

Before stating Theorem I.1, we recall a few definitions about quadratic spaces ( $W, S$ ) defined over $K$. Assume that $n=\operatorname{dim} W$ and that in diagonal form $S$ is $\operatorname{diag}\left(a_{1}, \ldots, a_{n}\right)$ where $a_{i} \in K^{*}$ (the multiplicative group of nonzero elements in $K)$. Then one puts $\Delta(S)=(-1)^{n(n-1) / 2} a_{1} \cdots a_{n} \bmod \left(K^{*}\right)^{2}$. The invariant $c(S)$ is the cohomology class of a certain 2-cocycle of $\Gamma$ in $\bar{K}^{*}$ and is defined in the proof of Theorem I.1. It can be shown [4] that the invariants $\operatorname{dim}, \Delta$, and $c$ are enough to determine $S$ if $K$ is a nonarchimedean local field.

THEOREM I.1. Let $G_{1}$ and $G$ be simply connected algebraic groups defined over $K$ (char $K=0$ ) and assume that there is a $\bar{K}$-isomorphism $f: G_{1} \rightarrow G$ such that $f^{-\sigma} \circ f=I_{g_{\sigma}}$ for each $\sigma \in \Gamma$. Define elements $c_{\sigma, \tau} \in Z\left(G_{1}\right)$ by setting $c_{\sigma, \tau}=g_{\sigma, \tau}^{-1} g_{\sigma}^{\tau} g_{\tau}$. Let $(V, \rho, B)$ be an orthogonal representation of $G$ defined over $K$ and assume that 
each $(\rho \circ f)\left(c_{\sigma, \tau}\right)$ is 1 . Then there is an orthogonal representation $\left(V_{1}, \rho_{1}, B_{1}\right)$ of $G_{1}$ defined over $K$ such that $\rho_{1} \sim \rho \circ f$ and $B_{1}$ is related to $B$ as follows: $\operatorname{dim} V_{1}=\operatorname{dim} V$, $\Delta\left(B_{1}\right)=\Delta(B)$, and $c\left(B_{1}\right)=c(B)\left(\rho_{s}\left(c_{\sigma, \tau}\right)\right)$ where $\rho_{s}: G_{1} \rightarrow \operatorname{Spin}(B)$ and $\pi \circ \rho_{s}=\rho \circ f$.

Proof. As before, we set $A_{\sigma}=(\rho \circ f)\left(g_{\sigma}^{-1}\right)$ and $\bar{A}_{\sigma}=\rho_{s}\left(g_{\sigma}^{-1}\right)$. Since $A_{\sigma}^{\tau} A_{\tau}=A_{\sigma \tau}$, there is an element $X \in G L(V)$ such that $A_{\sigma}=X^{-\sigma} X$. Using $X$, we set $\rho_{1}=X(\rho \circ f) X^{-1}$ and $B_{1}={ }^{t} X^{-1} B X^{-1}$. It is easy to check that $\rho_{1}$ is defined over $K$ and that the image of $G_{1}$ under $\rho_{1}$ preserves $B_{1}$ which is also defined over $K$. Also, since $A_{\sigma} \in S O(V, B),(\operatorname{det} X)^{\sigma}(\operatorname{det} X)^{-1}=1$ for all $\sigma \in \Gamma$ and so $(\operatorname{det} X) \in K^{*}$. Hence, $\Delta\left(B_{1}\right)=\Delta(B)$.

Finally, it is necessary to compute $c\left(B_{1}\right)$. To do this, we look at the Clifford algebra $C(B)$ of $B$. (If $\operatorname{dim} V \equiv 1(2)$, we really need $C^{+}(B)$, the set of even elements of $C(B)$, but we write $C(B)$ to avoid some notational clumsiness.) Let $h: C(B)$ $\rightarrow M(t, \bar{K})$ be an isomorphism of $C(B)$ onto a total matrix algebra. For each $\sigma \in \Gamma$, there is $Y_{\sigma} \in G L(t, \bar{K})$ such that $h^{\sigma}(x)=Y_{\sigma} h(x) Y_{\sigma}^{-1}$ for all $x \in C(B)$. The system $\left\{Y_{\sigma}\right\}$ satisfies the relation $Y_{\sigma}^{\tau} Y_{\tau}=b_{\sigma, \tau} Y_{\sigma, \tau}$ with $b_{\sigma, \tau} \in \bar{K}^{*}$ and, by definition, the cohomology class of the 2-cocycle $b_{\sigma, \tau}$ is $c(B)$.

The map $X^{-1}:\left(V_{1}, B_{1}\right) \rightarrow(V, B)$ is a quadratic space isomorphism and induces a mapping $X^{-1}: C\left(B_{1}\right) \rightarrow C(B)$. (In the following when we write $X^{-1}$, we shall always mean the mapping of the Clifford algebras.) The composite map $H=h \circ X^{-1}$ gives an isomorphism of $C\left(B_{1}\right)$ with a total matrix algebra. We now determine the corresponding 2-cocycle. For each $\sigma \in \Gamma, H^{\sigma} \circ H^{-1}=I_{N_{\sigma}}$ where $N_{\sigma}=Y_{\sigma} h\left(\bar{A}_{\sigma}\right)$. From this it follows that $N_{\sigma}^{\tau} N_{\tau}=b_{\sigma, \tau} \rho_{s}\left(c_{\sigma, \tau}\right) N_{\sigma \tau}$ and our theorem is proved.

It is not difficult to reduce the general case where $G_{1}$ is not simply connected to the case above. For it is known that there are simply connected covering groups $\left(\bar{G}_{1}, p_{1}\right)$ and $(\bar{G}, p)$ of $G_{1}$ and $G$ respectively which are defined over $K$. Then, it also can be shown that there is a $\bar{K}$-isomorphism $\bar{f}: \bar{G}_{1} \rightarrow \bar{G}$ such that for each $\sigma \in \Gamma, \bar{f}^{-\sigma} \circ \bar{f}=I_{h_{\sigma}}$; here, $h_{\sigma}$ is an element in $\bar{G}_{1}$ such that $p_{1}\left(h_{\sigma}\right)=g_{\sigma}$. In the statement of Theorem I.1, $G$ is replaced by $\bar{G}, \rho$ by $\rho \circ p, g_{\sigma}$ by $h_{\sigma}$, and so on.

1.3. Problem 2. In this section, we consider the case where $K \#$ is a quaternion division algebra $(\beta, \gamma)$ and we begin by summarizing some results which can be found in [7, p. 235]. The algebra $K \#$ has a basis $\left(1, x_{1}, x_{2}, x_{1} x_{2}\right)$ over $K$ such that $x_{1}^{2}=\beta, x_{2}^{2}=\gamma$, and $x_{1} x_{2}=-x_{2} x_{1}$. The elements $\beta$ and $\gamma$ are in $K^{*}$ and we assume that the equation $\beta X^{2}+\gamma Y^{2}=1$ has no solution $(X, Y)$ in $K$. An isomorphism $M: K \# \rightarrow M(2, \bar{K})$ is given by

$$
M\left(Y_{0}+Y_{1} x_{1}+Y_{2} x_{2}+Y_{3} x_{1} x_{2}\right)=\left(\begin{array}{cc}
Y_{0}+Y_{1} \beta^{1 / 2} & \gamma\left(Y_{2}+Y_{3} \beta^{1 / 2}\right) \\
Y_{2}-Y_{3} \beta^{1 / 2} & Y_{0}-Y_{1} \beta^{1 / 2}
\end{array}\right)
$$

$M$ is defined over $L=K\left(\beta^{1 / 2}\right)$ and if we set $\mathrm{Gal}(L / K)=\{1, \sigma\}$, then $M^{\sigma}(x)$ $=M\left(n_{\sigma}^{-1} x n_{\sigma}\right)$ for all $x \in K \#$ where $n_{\sigma}=x_{2}$. There is a canonical involution $x \rightarrow \bar{x}$ 
of the first kind on $K \#$, namely, if $x=Y_{0}+Y_{1} x_{1}+Y_{2} x_{2}+Y_{3} x_{1} x_{2}$, then $\bar{x}=Y_{0}$ $-Y_{1} x_{1}-Y_{2} x_{2}-Y_{3} x_{1} x_{2}$. Setting

$$
J=\left(\begin{array}{rr}
0 & -1 \\
1 & 0
\end{array}\right)
$$

we see that $M(\bar{x})=J^{-1}{ }^{t} M(x) J$ for all $x \in K$. Furthermore, ${ }^{t} J=-J$.

Now we return to the situation in Problem 2 and assume that $K \#$ is a quaternion division algebra. If $e_{i j}$ are matrix units in $K \#$, then, considering $V_{1} e_{11}$ as a vector space over $\bar{K}$, there is a $\bar{K}$-isomorphism $f_{1}: V \rightarrow V_{1} e_{11}$ defined over $L$ such that

$$
R_{n_{\sigma}}=a_{\sigma} f_{1} \circ A_{\sigma}^{-1} \circ f_{1}^{-\sigma}
$$

where $R_{n_{\sigma}}: V_{1} e_{22} \rightarrow V_{1} e_{11}$ is given by $R_{n_{\sigma}}(v)=v n_{\sigma}$ for all $v \in V_{1} e_{22}$. The element $a_{\sigma}$ is in $\bar{K}^{*}[7$, p. 229].

Define $B_{11}$ on $V_{1} e_{11}$ so that $f_{1}$ is a quadratic space isomorphism and set $B_{i j}(v, w)=B_{11}\left(v e_{i 1}, w e_{j 1}\right)$ for all $v, w \in V_{1}$ and $i, j=1,2,3,4$. Then the form $F$ is defined by the formula [7, p. 233]

$$
J M(F(v, w))=\left(B_{i j}(v, w)\right) .
$$

$F$ is skew-hermitian if $B$ is orthogonal.

LEMMA I.3. In formula (3), $a_{\sigma}^{2}=-\gamma$.

Proof. First we show that $-\gamma B_{1}^{\sigma}\left(v n_{\sigma}^{-1}, y n_{\sigma}^{-1}\right)=B_{11}(v, w)$ for all $v, w \in V_{1}$. This is done by applying $\sigma$ to (4) and remembering that $F$ is defined over $K, M^{\sigma} \circ I_{n_{\sigma}}=M$, and $n_{\sigma}=\gamma e_{12}+e_{21}$.

Using this result we are able to prove the lemma. Again we use (3) and the fact that $A_{\sigma} \in S O(V, B)$. For choosing $v$ to be $K$-rational in $V_{1}$, such that $B_{22}(v)$ $=B_{22}(v, v) \neq 0$, we have: $B_{11}\left(R_{n_{\sigma}}\left(v e_{22}\right)\right)=B_{11}\left(v e_{21}\right)=B_{22}(v)$. But also $a_{\sigma}^{-2} B_{11}\left(R_{n_{\sigma}}\left(v e_{22}\right)\right)$ $=B_{11}\left(f_{1} \circ A_{\sigma}^{-1} \circ f_{1}^{-\sigma}\left(v e_{22}\right)\right)=\left(B\left(f_{1}^{-1}\left(v e_{11}\right)\right)\right)^{\sigma}=\left(B_{11}\left(v e_{11}\right)\right)^{\sigma}=B_{11}\left(v n_{\sigma} e_{11} n_{\sigma}^{-1}\right)$ which by the first part of this lemma is just $(-\gamma)^{-1} B_{11}\left(v n_{\sigma} e_{11}\right)=(-\gamma)^{-1} B_{11}\left(v e_{21}\right)$ $=(-\gamma)^{-1} B_{22}(v)$ and the lemma is complete.

Before stating Theorem I.2, we again review some fundamental definitions. For a skew-hermitian form $F$ on a space $V_{1}$ over $K \#$, Tsukamoto [8] has determined a complete set of invariants when $K$ is a nonarchimedean local field such that $\left[K^{*}:\left(K^{*}\right)^{2}\right]>2$. The invariants are $\operatorname{dim} V_{1}$ and $\delta(F)$. This last invariant is defined in the following way: let $\left\{v_{1}, \ldots, v_{m}\right\}$ be an orthogonal basis defined over $K$ of $V_{1}$ over $K \#$. Since $F$ is skew-hermitian, $F\left(v_{i}, v_{i}\right)=x_{i}=-\bar{x}_{i}$ for some $x_{i} \in K \#$. But $x_{i}^{2}=a_{i} \in K^{*}$ and we set $\delta(F)=a_{1} \cdots a_{m} \bmod \left(K^{*}\right)^{2}$.

Theorem I.2. Let $G_{1}$ and $G$ be semisimple algebraic groups defined over $K$ (char $K=0$ ) and assume that there is a $\bar{K}$-isomorphism $f_{1}: G_{1} \rightarrow G$ such that $f^{-\sigma} \circ f=I_{g_{\sigma}}$ for each $\sigma \in \Gamma$. Let $(V, \rho, B)$ be an orthogonal representation of $G$ defined over $K$ and let $\left(V_{1} / K \#, \rho_{1}, F\right)$ be a skew-hermitian representation of $G$ defined over $K$ where $K \#$ is a quaternion division algebra over $K$. Assume also that there is an 
absolutely irreducible representation $\theta_{1}$ : End $\left(V_{1} / K \#\right) \rightarrow$ End $(V)$ defined over $\bar{K}$ such that $\theta_{1}\left(\rho_{1}(g)\right)=(\rho \circ f)(g)$ for each $g \in G_{1}$. Then the invariants of $F$ are as follows: $\operatorname{dim} V_{1}=\frac{1}{2} \operatorname{dim} V$ and $\delta(F)=\Delta(B)$.

Proof. The dimension formula follows from the existence of $f_{1}$ in (3). To prove the relation on discriminants, let $\left\{v_{1}, \ldots, v_{m}\right\}$ be an orthogonal basis of $F$ defined over $K$. Then $E=\left\{v_{1} e_{11}, \ldots, v_{m} e_{11}, v_{1} e_{21}, \ldots, v_{m} e_{21}\right\}$ is a basis for $V_{1} e_{11}$ and $\delta(F)=(-1)^{m} \operatorname{det}\left(B_{11}, E\right)$. By this last term we mean the determinant of $B_{11}$ in the basis $E$.

Let $\left\{x_{1}, \ldots, x_{2 m}\right\}$ be a basis of $V$ defined over $K$ and let $P$ be the matrix of $f^{-1}(E)$ with respect to $\left\{x_{i}\right\}$. Then $\delta(F)=(-1)^{m} \operatorname{det}\left(B,\left\{x_{i}\right\}\right) \cdot(\operatorname{det} P)^{2}$. Hence, $(\operatorname{det} P)^{2} \in K^{*}$. If we can show that $\operatorname{det} P \in K^{*}$, we are done. Stated differently, it remains to be proved that $(\operatorname{det} P)^{\sigma}(\operatorname{det} P)^{-1}=1$ where $\operatorname{Gal}(L / K)=\{1, \sigma\}$.

To prove this statement, we compute determinants of both sides of (3). The matrix of $R_{n^{-1}}(E)$ in the basis $E^{\sigma}=\left\{v_{1} e_{22}, \ldots, v_{m} e_{22}, \gamma v_{1} e_{12}, \ldots, \gamma v_{m} e_{12}\right\}$ is

$$
\left(\begin{array}{cc}
0 & 1_{m} \\
\gamma^{-1} 1_{m} & 0
\end{array}\right)
$$

and has determinant $(-\gamma)^{-m}$. So, by (3), it follows that $(\operatorname{det} P)^{\sigma}(\operatorname{det} P)^{-1}$ $=(-\gamma)^{-m} a_{\sigma}^{2 m}=(-\gamma)^{-m}(-\gamma)^{m}$, by Lemma I.3, and we have proved the theorem.

1.4. Steinberg groups. In this brief section, we look at the results in this part from a slightly different viewpoint, namely that of Steinberg groups. A group $G$ defined over $K$ is called Steinberg if there is a Borel subgroup of $G$ which is also defined over $K$. It is known that if $G_{1}$ is a connected semisimple group defined over $K$, then there is a Steinberg group $G$ defined over $K$ and a $\bar{K}$-isomorphism $f: G_{1} \rightarrow G$ of inner type. In this case, the cohomology class of $c_{\sigma, \tau}$ is independent of $f$ and is denoted by $\gamma_{K}\left(G_{1}\right)$. This last invariant has been studied by Satake [6], [7].

The division algebra associated with an irreducible representation of a Steinberg group is always trivial, i.e., is the underlying field [7, p. 241]. Hence, in terms of Steinberg groups, Theorems I.1 and I.2 say that to determine the form on a representation of $G_{1}$ it is enough to know the form on the corresponding representation of the Steinberg group $G$ associated with $G_{1}$. Of course, for absolutely simple groups $G_{1}$, the associated Steinberg group $G$ will always be the corresponding Chevalley group except possibly when $G_{1}$ is of type $A_{n}, D_{n}$, or $E_{6}$. In Part II, we shall study these three cases and show how orthogonal representations of Steinberg and Chevalley groups are related.

\section{PART II}

2.1. The group $G^{*}$. Throughout this section, let $G$ be a semisimple Chevalley group defined over $K(\operatorname{char} K=0)$ and let $T$ be a maximal split torus in $G$ defined over $K$. Denote by $\Delta=\left\{\alpha_{1}, \ldots, \alpha_{n}\right\}$ the corresponding fundamental root system. 
The automorphism group of $G$ is the semidirect product of a finite group $\Theta$ and the inner automorphisms of $G$. We choose $\Theta$ in such a way that for each $\theta \in \Theta, \theta$ is defined over $K, \theta(T)=T$, and $\theta(\Delta)=\Delta$. We define an algebraic group $G^{*}$ to be $G \cdot \Theta$, the semidirect product of $G$ and $\Theta$ where group multiplication is given in the following way: $\left(g_{1} \theta_{1}\right)\left(g_{2}, \theta_{2}\right)=\left(g_{1} \theta_{1}\left(g_{2}\right), \theta_{1} \theta_{2}\right)$. In what follows, we consider $G$ as a subgroup of $G^{*}$. By our choice of $\Theta$, both are algebraic groups defined over $K$.

Lemma II.1. Let $\rho: G \rightarrow G L(V)$ be an absolutely irreducible representation of $G$ defined over $K$. Then there exists a representation $\rho^{*}: G^{*} \rightarrow G L(V)$ defined over $\bar{K}$ such that $\rho^{*} \mid G=\rho$ if and only if there is a homomorphism $\theta \rightarrow A_{\theta}$ of $\Theta$ to $G L(V)$ such that $\rho(\theta(g))=A_{\theta} \rho(g) A_{\theta}^{-1}$ for all $g \in G$.

Proof. If $\rho^{*}$ exists, set $\rho^{*}(1, \theta)=A_{\theta}$. Then $\rho^{*}\left[(1, \theta)(g, 1)\left(1, \theta^{-1}\right)\right]=A_{\theta} \rho(g) A_{\theta}^{-1}$ and is also $\rho^{*}((\theta(g), 1))=\rho(\theta(g))$.

Conversely, if such $A_{\theta}$ exist, define $\rho^{*}(g, \theta)=\rho(g) A_{\theta}$. It is easy to check that $\rho^{*}$ becomes a homomorphism and so the lemma is proved.

Corollary. Assume that $\Theta$ is a cyclic group generated by $\theta$. Then $\rho^{*}$ exists if and only if $\rho \circ \theta \sim \rho$.

Proof. Assume that $\theta^{r}=1$ and $\rho \circ \theta=A_{\theta} \rho A_{\theta}^{-1}$. It is easy to see that $A_{\theta}^{r}=a 1$ for some $a \in \bar{K}^{*}$ and modifying $A_{\theta}$ we can assume $A_{\theta}^{r}=1$. This completes the proof.

2.2. The groups $A_{n}, D_{n}$, and $E_{6}$. In this section, we shall take a closer look at the group $G^{*}$ when $G$ is a Chevalley group of type $A_{n}, D_{n}$, or $E_{6}$. In particular, let $(V, \rho, B)$ be an orthogonal representation of $G$ defined over $K$ with highest weight $\lambda$. We shall give conditions on $\lambda$ in order that $\rho^{*}: G^{*} \rightarrow G L(V)$ exists; furthermore, in each case we shall show that $\rho^{*}$ can be chosen to be defined over $K$ and $\rho^{*}: G^{*} \rightarrow O(V, B)$.

Lemma II.2. Let $G$ be a Chevalley group of type $A_{n}$ defined over $K(\operatorname{char} K=0)$ and let $(V, \rho, B)$ be an orthogonal representation of $G$ defined over $K$. Then $\rho^{*}: G^{*} \rightarrow O(V, B)$ exists and is defined over $K$. Furthermore, if $\operatorname{dim} V \equiv 1(2), \rho^{*}$ can be chosen so that $\rho^{*}: G^{*} \rightarrow S O(V, B)$.

Proof. For easy reference, the proof is divided into small sections.

(i) The group $\Theta$ is of order 2 and is generated by $\theta$ where $\theta\left(\alpha_{r}\right)=\alpha_{n-r+1}$. If $\lambda=\sum_{r=1}^{n} m_{r} \alpha_{r}$ with $m_{r} \in \boldsymbol{Q}, m_{r} \geqq 0$, then $\rho \circ \theta \sim \rho$ if and only if $m_{r}=m_{n-r+1}$. But it is known [3, p. 196] that all orthogonal representations of $A_{n}$ have this property and also that each $m_{r} \in Z$. Since $\rho$ and $\rho \circ \theta$ are both defined over $K$, there is an $A \in G L(V, K)$ such that $A \rho(g)=\rho(\theta(g)) A$. Let $x$ be a $K$-rational highest weight vector in $V$. Since $\theta \lambda=\lambda$, it is easy to see that $A x$ is also a $K$-rational highest weight vector. Hence, $A x=a x$ for some $a \in K^{*}$ and $A^{2}=a^{2}$. Set $A_{\theta}=a^{-1} A$; then $A_{\theta} \in G L(V, K), A_{\theta} \rho(g)=\rho(\theta(g)) A_{\theta}$ for all $g \in G$, and $A_{\theta}^{2}=1$. If $\operatorname{dim} V \equiv 1$ (2), we may assume that $\operatorname{det} A_{\theta}=1$, multiplying $A_{\theta}$ by -1 if necessary. We also note that $A_{\theta} x=e x$ where $e^{2}=1$. Next, we shall show that $A_{\theta}$ is in $O(V, B)$. 
(ii) Let $W=N(T) / T$ be the Weyl group of $G$. It is known that there is an element $w$ in $W$ such that $w(\Delta)=-\Delta$, i.e. $w\left(\alpha_{r}\right)=-\alpha_{n-r+1}$. Choose a representative $g$ in $N(T)$ for w, i.e. $w=g T$. The element $\theta(g)$ is also in $N(T)$ and it is easy to see that $I_{\theta(g)}=\theta \circ I_{g} \circ \theta=I_{g}$ on $T$. (It is enough to check that the induced mappings on $\Delta$ agree.) Hence, there is a $t$ in $T$ such that $\theta(g)=g t$. Applying $\theta$ again to this equation we get

$$
t \theta(t)=1 .
$$

(iii) Next, we show that $B(x, \rho(g) x) \neq 0$. If $x_{1}$ and $x_{2}$ are weight vectors in $V$ corresponding to weights $\lambda_{1}$ and $\lambda_{2}$, respectively, then for $t$ in $T, B\left(x_{1}, x_{2}\right)$ $=B\left(\rho(t), x_{1}, \rho(t) x_{2}\right)=\lambda_{1}(t) \lambda_{2}(t) B\left(x_{1}, x_{2}\right)$. So $B\left(x_{1}, x_{2}\right)=0$ except possibly when the character $\lambda_{1}+\lambda_{2}$ is 0 . (We use additive notation on the character module of $T$.) In the case above, the highest weight space has dimension 1 and so if $\rho(g) x$ has weight $-\lambda$, then we are done with (iii). But this follows from the facts that $g \in N(T)$ and $I_{g}(\lambda)=-\lambda$.

Since ${ }^{t} A_{\theta} B A_{\theta}$ is also invariant under $\rho(G)$, there is $a_{\theta} \in K^{*}$ such that ${ }^{t} A_{\theta} B A_{\theta}=a_{\theta} B$. In particular $0 \neq a_{\theta} B(x, \rho(g) x)=B\left(A_{\theta} \cdot x, A_{\theta} \rho(g) x\right)=B\left(A_{\theta} x, \rho(\theta(g)) A_{\theta} x\right)=B(x, \rho(g t) x)$ $=\lambda(t) B(. x, \rho(g) x)$. Hence, $a_{\theta}=\lambda(t)$. The map $\theta \rightarrow a_{\theta}$ is a homomorphism and so $a_{\theta}^{2}=1$, i.e. $\lambda(t)^{2}=1$, a result which can also be seen by applying $\lambda$ to (5).

(iv) Finally, we show that $\lambda(t)=1$. If $n \equiv 0$ (2), this follows immediately. For by (5), $\left(\alpha_{r}+\alpha_{n-r+1}\right)(t)=1$; but $\lambda$ is an integral combination of such terms. If $n \equiv 1$ (2), then it is enough to show that $\alpha_{r}(t)=1$ where $r=\frac{1}{2}(n+1)$. We saw that ${ }^{t} A_{\theta} B A_{\theta}$ $=\lambda(t) B$. In particular, if $\operatorname{dim} V \equiv 1(2)$, then $\lambda(t)=1$ (as can be seen by taking determinants). But for $n \equiv 1$ (2), the representation with highest weight $\lambda=\alpha_{1}+\alpha_{2}$ $+\cdots+\alpha_{n}$ is orthogonal and has dimension $n(n+2)$ which is odd. Hence, $\lambda(t)=\alpha_{r}(t)=1$ and the lemma is proved.

We have proved this lemma in such generality so that the proof will apply in the cases $D_{n}$ and $E_{6}$. We indicate below the way in which this happens.

Lemma II.3. Let $G$ be a Chevalley group of type $D_{n}(n \neq 4)$ defined over $K$ (char $K=0)$ and let $(V, \rho, B)$ be an orthogonal representation of $G$ defined over $K$ with highest weight $\lambda=\sum_{r=1}^{n} m_{r} \alpha_{r}$. Then $\rho^{*}: G^{*} \rightarrow O(V, B)$ exists and is defined over $K$ if and only if $m_{n}=m_{n-1}$. Furthermore, if $\operatorname{dim} V \equiv 1$ (2), $\rho^{*}$ can be chosen so that $\rho^{*}: G^{*} \rightarrow S O(V, B)$.

Proof. We take $G=S O(2 n)$, the special orthogonal group on a $2 n$-dimensional vector space $W$ defined over $K$. Let $\left\{e_{1}, \ldots, e_{2 n}\right\}$ be a $K$-rational basis of weight vectors where $e_{i}$ has weight $\lambda_{i}$ and $e_{n+i}$ has weight $-\lambda_{i}$ for $i=1, \ldots, n$. A fundamental root system $\left\{\alpha_{1}, \ldots, \alpha_{n}\right\}$ is given by $\alpha_{1}=\lambda_{1}-\lambda_{2}, \ldots, \alpha_{n-1}=\lambda_{n-1}-\lambda_{n}$, and $\alpha_{n}=\lambda_{n-1}+\lambda_{n}$. Define a linear transformation $J \in O(2 n)$ by $J e_{r}=e_{r}, r \neq n, 2 n$, $J e_{n}=e_{2 n}$, and $J e_{2 n}=e_{n}$. Then $\operatorname{det}(J)=-1$.

(i) The group $\Theta$ is of order 2 and is generated by $\theta$ where $\theta\left(\alpha_{n-1}\right)=\alpha_{n}$. If $\lambda=\sum_{r=1}^{n} m_{r} \alpha_{r}$ with $m_{r} \in Q, m_{r} \geqq 0$, then $\rho \circ \theta \sim \rho$ if and only if $m_{n}=m_{n-1}$. It is easy to see that $\theta=I_{j}$. Hence, $G^{*}$ may be identified with $O(2 n)$. 
(ii) The element $w=g T$ is given in the following way: if $n \equiv 1$ (2), $g e_{r}=e_{r+n}$ for $r=1, \ldots, n-1, g e_{n}=e_{n}, g e_{2 n}=e_{2 n}$, and $g^{2}=1$. If $n \equiv 0$ (2), $g e_{r}=e_{r+n}$ for $r=1, \ldots, n$ and $g^{2}=1$. In either case, $\theta(g)=J g J=g$ and so $t=1$. The lemma now follows immediately.

The case $D_{4}$ is complicated by the fact that $\Theta=S_{3}$, the symmetric group on 3 elements. We postpone our study of it, looking first at $E_{6}$.

Lemma 11.4. Let $G$ be a Chevalley group of type $E_{6}$ defined over $K(\operatorname{char} K=0)$ and let $(V, \rho, B)$ be an orthogonal representation of $G$ defined over $K$. Then $\rho^{*}$ : $G^{*} \rightarrow O(V, B)$ exists and is defined over $K$. Furthermore, if $\operatorname{dim} V \equiv 1(2), \rho^{*}$ can be chosen so that $\rho^{*}: G^{*} \rightarrow S O(V, B)$.

Proof. The group $G$ has the following Dynkin diagram:

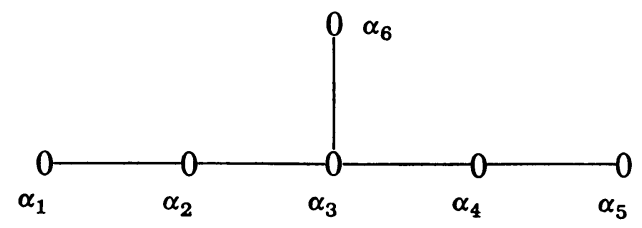

(i) The group $\Theta$ is of order 2 and is generated by $\theta$ where $\theta\left(\alpha_{1}\right)=\alpha_{5}, \theta\left(\alpha_{2}\right)=\alpha_{4}$, $\theta\left(\alpha_{3}\right)=\alpha_{3}$, and $\theta\left(\alpha_{6}\right)=\alpha_{6}$. If $\lambda=\sum_{r=1}^{6} m_{r} \alpha_{r}$ with $m_{r} \in Q, m_{r} \geqq 0$, then $\rho \circ \theta \sim \rho$ if and only if $m_{1}=m_{5}$ and $m_{2}=m_{4}$. But it is known [3, p. 202] that all orthogonal representations of $E_{6}$ have this property and also that each $m_{r} \in \boldsymbol{Z}$.

(ii) The element $w$ is given by: $w\left(\alpha_{1}\right)=-\alpha_{5}, w\left(\alpha_{2}\right)=-\alpha_{4}, w\left(\alpha_{3}\right)=-\alpha_{3}$, and $w\left(\alpha_{6}\right)=-\alpha_{6}$.

(iv) We know that $\lambda$ is an integral combination of $\alpha_{1}+\alpha_{5}, \alpha_{2}+\alpha_{4}, \alpha_{3}$, and $\alpha_{6}$. From (5), it follows that $\left(\alpha_{1}+\alpha_{5}\right)(t)=1,\left(\alpha_{2}+\alpha_{4}\right)(t)=1$ and $\alpha_{3}(t)^{2}=\alpha_{6}(t)^{2}=1$. Hence, it only remains to be shown that $\alpha_{3}(t)=\alpha_{6}(t)=1$. The representation with highest weight $\lambda=2\left(\alpha_{1}+\alpha_{5}\right)+4\left(\alpha_{2}+\alpha_{4}\right)+6 \alpha_{3}+3 \alpha_{6}$ is orthogonal and has odd dimension. But then $\lambda(t)=\alpha_{6}(t)=1$. Similarly, the representation with highest weight $\lambda=5\left[\left(\alpha_{1}+\alpha_{5}\right)+2\left(\alpha_{2}+\alpha_{4}\right)+3 \alpha_{3}+2 \alpha_{6}\right]$ is orthogonal and has odd dimension. Hence, $\alpha_{3}(t)=1$ and the proof of the lemma is completed.

Lemma II.5. Let $G$ be a Chevalley group of type $D_{4}$ defined over $K\left(K=Q_{\Downarrow}\right)$ and let $(V, \rho, B)$ be an orthogonal representation of $G$ defined over $K$ with highest weight $\lambda=m_{1} \alpha_{1}+m_{2} \alpha_{2}+m_{3} \alpha_{3}+m_{4} \alpha_{4}$. Then $\rho^{*}: G^{*} \rightarrow O(V, B)$ exists and is defined over $K$ if and only if $m_{1}=m_{3}=m_{4}$. Furthermore, if $\operatorname{dim} V \equiv 1$ (2), $\rho^{*}$ can be chosen so that $\rho^{*}: G^{*} \rightarrow \operatorname{SO}(V, B)$.

Proof. The group $G$ has the following Dynkin diagram:

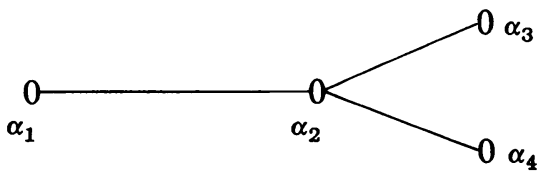


(i) The group $\Theta$ is of order 6 and is the symmetric group on $\left\{\alpha_{1}, \alpha_{3}, \alpha_{4}\right\}$. We distinguish two elements $\theta$ and $\psi$ in $\Theta$. The element $\theta$ has order 2 and is defined by $\theta\left(\alpha_{3}\right)=\alpha_{4}$ and the element $\psi$, having order 3 , is defined by $\psi\left(\alpha_{1}\right)=\alpha_{3}, \psi\left(\alpha_{3}\right)=\alpha_{4}$, and $\psi\left(\alpha_{4}\right)=\alpha_{1}$. If $\lambda=m_{1} \alpha_{1}+m_{2} \alpha_{2}+m_{3} \alpha_{3}+m_{4} \alpha_{4}$, it follows that a necessary condition for $\rho^{*}: G^{*} \rightarrow G L(V)$ to exist is that $m_{1}=m_{3}=m_{4}$. We show now that these equalities are also sufficient. For let $x$ be a $K$-rational highest weight vector of $\rho$. Then, as in the proof for $A_{n}$, there are elements $A_{\theta}, A_{\psi} \in G L(V, K)$ such that $A_{\theta}^{2}=A_{\psi}^{3}=1, A_{\theta} \rho(g)=\rho(\theta(g)) A_{\theta}$ and $A_{\psi} \rho(g)=\rho(\psi(g)) A_{\psi}$ for all $g \in G, A_{\theta} x=x$, and $A_{\psi} x=x$. The defining relations for $S_{3}$ are $\theta^{2}=\psi^{3}=1$ and $\theta \psi \theta=\psi^{2}$. Hence, we need to show that $A_{\theta} A_{\psi} A_{\theta}=A_{\psi}^{2}$. But since

$$
\rho\left(\psi^{2}(g)\right)=A_{\psi}^{2} \rho(g) A_{\psi}^{-2}=\left(A_{\theta} A_{\psi} A_{\theta}\right) \rho(g)\left(A_{\theta} A_{\psi} A_{\theta}\right)^{-1}
$$

it follows that there exists $a \in K^{*}$ such that $A_{\psi}^{2}=a A_{\theta} A_{\psi} A_{\theta}$. Applying both sides to $x$, we see that $a=1$ and this part of the lemma is proved. It should be noticed, also, that we can assume $\operatorname{det} A_{\theta}=1$ if $\operatorname{dim} V \equiv 1$ (2).

As in Lemma II.3, it can be shown that $A_{\theta} \in O(V, B)$. Therefore, if we can show that $A_{\psi}$ is in $O(V, B)$, the proof will be complete. As a matter of fact, since the mapping $\psi \rightarrow A_{\psi}$ gives a homomorphism of the group of order 3 generated by $\psi$, if $A_{\psi} \in O(V, B)$, then $A_{\psi} \in S O(V, B)$.

We know that ${ }^{t} A_{\psi} B A_{\psi}=a_{\psi} B$ where $a_{\psi} \in K^{*}$ and $a_{\psi}^{3}=1$. But since $G$ is a Chevalley group, we may assume that $K=Q$ and then $a_{\psi}$ must be 1 . This completes the proof of the lemma.

To conclude this section, we prove a result about the Clifford algebra $C(B)$ of $B$ which will be useful when we return to Problem 1. As above, the set of even elements in $C(B)$ will be denoted by $C^{+}(B)$.

Lemma II.6. Let $G$ be a Chevalley group of type $A_{n}, D_{n}$, or $E_{6}$ defined over $K$ (char $K=0)$ and let $\theta \in \Theta$ be an element of order 2 . Let $(V, \rho, B)$ be an orthogonal representation of $G$ defined over $K$ and assume that $\rho^{*}: G^{*} \rightarrow O(V, B)$ exists and is defined over $K$. Then there is an element $\bar{A}_{\theta}$ in $C^{+}(B)$ if $\operatorname{det} A_{\theta}=1$ or in $C(B)$ if $\operatorname{det} A_{\theta}=-1$ satisfying the following conditions:

(i) $\bar{A}_{\theta} x \bar{A}_{\theta}^{-1}=A_{\theta} x$ for all $x \in V$.

(ii) $A_{\theta}(\operatorname{Spin}(B)) A_{\theta}^{-1}=\operatorname{Spin}(B)$.

Proof. Since $A_{\theta}=\rho^{*}(\theta)$ is defined over $K, A_{\theta}^{2}=1$, and $A_{\theta} \in O(V, B)$, the spaces $V^{+}=\left\{x \in A_{\theta} \mid x=x\right\}$ and $V^{-}=\left\{x \in V \mid A_{\theta} x=-x\right\}$ are defined over $K$, span $V$, and are perpendicular. Let $\left\{e_{1}, \ldots, e_{r}\right\}$ and $\left\{e_{r+1}, \ldots, e_{n}\right\}$ be orthogonal bases of $V^{+}$ and $V^{-}$, respectively, which are defined over $K$.

If $\operatorname{det} A_{\theta}=1$ (i.e., $n-r \equiv 0$ (2)), we set $\bar{A}_{\theta}=e_{r+1} \cdots e_{n} \in C^{+}(B)$. If $\operatorname{det} A_{\theta}=-1$ (i.e., $n \equiv 0$ (2) and $n-r \equiv 1(2)$ ), we set $\bar{A}_{\theta}=e_{1} \cdots e_{r} \in C(B)$. In both cases it is easy to see that $\bar{A}_{\theta}$ has the desired properties and so the lemma is proved.

Corollary 1. Let $\rho_{s}: G \rightarrow$ Spin $(B)$ be such that $\pi \circ \rho_{s}=\rho$ where $\pi$ is the natural mapping from Spin $(B)$ onto $S O(V, B)$. Then $\rho_{s}(\theta(g))=\bar{A}_{\theta} \rho(s) \bar{A}_{\theta}^{-1}$ for all $g \in G$. 
Corollary 2. If det $A_{\theta}=1$, then $\bar{A}_{\theta}^{2}=\Delta^{-}$where $\Delta^{-}$is the discriminant of $B$ restricted to $V^{-}=\left\{x \in V \mid A_{\theta} x=-x\right\}$. If $\operatorname{det} A_{\theta}=-1$, then $\bar{A}_{\theta}^{2}=\Delta^{+}$where $\Delta^{+}$is the discriminant of $B$ restricted to $V^{+}=\left\{x \in V \mid A_{\theta} x=x\right\}$.

2.3. Problem 1. Having the above results in hand, we are now able to give solutions to Problems 1 and 2 if $f$ is not of inner type. As we saw in $\$ 1.4$, we have reduced Problem 1 to the case where $G_{1}$ is a Steinberg group of type $A_{n}, D_{n}$, or $E_{6}$ and $G$ is the corresponding Chevalley group.

Let $G$ be a semisimple Chevalley group defined over $K$ and let $\Theta$ be chosen as above. Steinberg groups are just $K$-forms associated with continuous 1-cocycles in $\Theta$. Indeed, let $\left\{\theta_{\sigma}\right\}$ be a continuous 1-cocycle in $\Theta$, i.e., $\theta_{\sigma} \theta_{\tau}=\theta_{\sigma \tau}$ for all $\sigma, \tau \in \Gamma$ and let $G_{1}$ be the associated $K$-form. Let $\Delta_{1}$ be a fundamental system in $G_{1}$ corresponding to $\Delta$. Then $\Delta_{1}^{\sigma}=\Delta_{1}$ for all $\sigma \in \Gamma$ and using this it can be shown that $G_{1}$ is Steinberg. Furthermore, there is a finite extension $K_{0}$ of $K$ over which $G_{1}$ is a Chevalley group. The elements $\sigma \in \mathrm{Gal}\left(K_{0} / K\right)$ correspond to $\theta_{\sigma} \in \Theta$ and if $\sigma \neq 1$, then $\theta_{\sigma} \neq 1$. This field $K_{0}$ is called the nuclear field of $G_{1}$ [5]. With the exception of $D_{4}, K_{0}$ is a quadratic extension of $K$. As we have seen, $\Theta=S_{3}$ is $G=D_{4}$ and $K=Q_{\mathfrak{p}}$. Hence, in this case, $\left[K_{0} / K\right]$ can be 2,3 , or 6 . In stating the next theorem, we use the notation introduced in $\$ 2.1$.

THEOREM II.1. Let $G_{1}$ be a Steinberg group of type $A_{n}, D_{n}(n \neq 4)$, or $E_{6}$ defined over $K(\operatorname{char} K=0)$, let $G$ be the corresponding Chevalley group defined over $K$, and let $f: G_{1} \rightarrow G$ be the isomorphism between $G_{1}$ and $G$ so that $f^{\sigma} \circ f^{-1}=\theta_{\sigma} \in \Theta$ for all $\sigma \in \Gamma$. Assume that $(V, \rho, B)$ is an orthogonal representation of $G$ defined over $K$ such that $\rho^{*}: G^{*} \rightarrow O(V, B)$ exists and is defined over $K$. Then there is an orthogonal representation $\left(V_{1}, \rho_{1}, B_{1}\right)$ of $G_{1}$ defined over $K$ such that $\rho_{1} \sim \rho \circ f$ and $B_{1}$ is related to $B$ as follows:

(i) $\operatorname{dim} V_{1}=\operatorname{dim} V$.

(ii) $\Delta\left(B_{1}\right)=\Delta(B)$ if $\operatorname{det}\left(\rho^{*}(\theta)\right)=1$ or $\Delta\left(B_{1}\right)=\alpha \Delta(B)$ if $\operatorname{det}\left(\rho^{*}(\theta)\right)=-1$ where $\alpha \in K^{*}$ is determined up to $\left(K^{*}\right)^{2}$ by the property that $K_{0}=K\left(\alpha^{1 / 2}\right)$ is the field fixed by $\left\{\sigma \in \Gamma \mid \theta_{\sigma}=1\right\}$.

(iii) $c\left(B_{1}\right)=c(B)\left(c_{\sigma, \tau}\right)$ where $c_{\sigma, \tau}=1$ unless $\theta_{\sigma}=\theta_{\tau}=\theta$. Then $c_{\sigma, \tau}=\Delta^{-}$if $\operatorname{det}\left(\rho^{*}(\theta)\right)$ $=1$ or $\Delta^{+}$if $\operatorname{det}\left(\rho^{*}(\theta)\right)=-1$.

Proof. This proof is a slight generalization of that for Theorem I.1. For $\sigma \in \Gamma$, $(\rho \circ f)^{\sigma}=\rho \circ \theta_{\sigma} \circ f=A_{\sigma}(\rho \circ f) A_{\sigma}^{-1}$ where $A_{\sigma}=\rho^{*}\left(\theta_{\sigma}\right)$. Hence, since $\rho^{*}$ is defined over $K, A_{\sigma}^{\tau} A_{\tau}=\rho^{*}\left(\theta_{\sigma} \theta_{\tau}\right)=\rho^{*}\left(\theta_{\sigma \tau}\right)=A_{\sigma \tau}$. There is an element $X \in G L(V)$ such that $A_{\sigma}=X^{-\sigma} X$ for all $\sigma \in \Gamma$. We put $\rho_{1}=X(\rho \circ f) X^{-1}$ and $B_{1}={ }^{t} X^{-1} B X^{-1}$. Then it is immediate that $\rho_{1} \sim \rho \circ f, \rho_{1}$ and $B_{1}$ are defined over $K, \rho_{1}$ preserves $B_{1}$, and $\operatorname{dim} V_{1}=\operatorname{dim} V$. Since $(\operatorname{det} X)^{\sigma}(\operatorname{det} X)^{-1}=\operatorname{det} A_{\sigma}=+1$ or -1 , the result on $\Delta\left(B_{1}\right)$ follows.

Finally, let $h: C(B) \rightarrow M(t, \bar{K})$ be an isomorphism of $C(B)$ onto a total matrix algebra. (Again, if $\operatorname{dim} V \equiv 1(2)$, we should write $C^{+}(B)$, but since nothing would 
change in the proof below, we do not distinguish these cases.) For $\sigma \in \Gamma$, there is $Y_{\sigma} \in G L(t, \bar{K})$ such that $h^{\sigma}(x)=Y_{\sigma} h(x) Y_{\sigma}^{-1}$ for all $x \in C(B)$. The system $\left\{Y_{\sigma}\right\}$ satisfies $Y_{\sigma}^{\tau} Y_{\tau}=b_{\sigma, \tau} Y_{\sigma \tau}$ with $b_{\sigma, \tau} \in \bar{K}^{*}$ and $c(B)=\left(b_{\sigma, \tau}\right)$.

Next, we use Lemma II.6. For setting $H=h \circ X^{-1}$ we have an isomorphism of $C\left(B_{1}\right)$ onto $M(t, \bar{K})$. For $\sigma \in \Gamma, H^{\sigma} \circ H^{-1}=I_{N_{\sigma}}$ where $N_{\sigma}=Y_{\sigma} h\left(\bar{A}_{\sigma}\right)$. Then $N_{\sigma}^{\tau} N_{\tau}$ $=b_{\sigma, \tau} c_{\sigma, \tau} N_{\sigma \tau}$. The elements $c_{\sigma, \tau}$ in $\bar{K}^{*}$ are defined by $\bar{A}_{\sigma} \bar{A}_{\tau}=c_{\sigma, \tau} \bar{A}_{\sigma \tau}$ and (iii) follows on applying Corollary 2 of Lemma II.6. Hence, the theorem is proved.

REMARK. In $\$ 2.2$, we saw that if $\rho \circ \theta \sim \rho$, then $\rho^{*}: G^{*} \rightarrow O(V, B)$ exists and is defined over $K$. Furthermore, if $\rho_{1}$ is a representation of $G_{1}$ defined over $K$ and if $\rho$ is the representation of $G$ defined over $K$ such that $\rho \sim \rho_{1} \circ f^{-1}$, then $\rho^{*}$ always exists since $\rho^{\sigma}=\rho$ implies $\rho_{1} \circ f^{-1} \circ \theta_{\sigma} \sim \rho_{1} \circ f^{-1}$. Therefore, Theorem II. 1 is a complete reduction to the Chevalley case of the problem of finding invariant orthogonal forms on representations of Steinberg groups of type $A_{n}, D_{n}(n \neq 4)$, and $E_{6}$.

Groups of type $D_{4}$ present no new problems and we shall only outline the results.

(1) If $\left[K_{0} / K\right]=2$, the situation is exactly as in Theorem II.1.

(2) If $\left[K_{0} / K\right]=3$, let $\tau \in \mathrm{Gal}\left(K_{0} / K\right)$ such that $\tau^{3}=1$. If $\rho^{*}: G^{*} \rightarrow O(V, B)$ exists, we have seen that $\operatorname{det}\left(A_{\tau}\right)=1$. Furthermore, we may find $\bar{A}_{\tau} \in \operatorname{Spin}(B)$ such that $\bar{A}_{\tau}^{3}=1$. So, $\operatorname{dim} V_{1}=\operatorname{dim} V, \Delta\left(B_{1}\right)=\Delta(B)$, and $c\left(B_{1}\right)=c(B)$.

(3) The case $\left[K_{0} / K\right]=6$ combines the results of (1) and (2). Indeed let $\sigma, \tau \in \mathrm{Gal}\left(K_{0} / K\right)$ have orders 2 and 3 respectively and let $\theta, \psi$ be the corresponding elements in $\Theta$. Then proceeding as in Theorem II.1, we get the following results: $\operatorname{dim} V_{1}=\operatorname{dim} V ; \Delta\left(B_{1}\right)=\Delta(B)$ if $\operatorname{det} \rho^{*}(\theta)=1$ and otherwise $\Delta\left(B_{1}\right)=\alpha \Delta(B)$ where $\alpha \in K^{*}$ is such that $\sigma\left(\alpha^{1 / 2}\right)=-\alpha^{1 / 2}$. Finally $c\left(B_{1}\right)=c(B) \cdot(2$-cocycle). The elements of this 2-cocycle are given in the following table:

$\begin{array}{lllllll} & 1 & \sigma & \tau & \tau^{2} & \sigma \tau & \sigma \tau^{2} \\ 1 & 1 & 1 & 1 & 1 & 1 & 1 \\ \sigma & 1 & \delta & 1 & 1 & \delta & \delta \\ \tau & 1 & 1 & 1 & 1 & 1 & 1 \\ \tau^{2} & 1 & 1 & 1 & 1 & 1 & 1 \\ \sigma \tau & 1 & \delta & 1 & 1 & \delta & \delta \\ \sigma \tau^{2} & 1 & \delta & 1 & 1 & \delta & \delta\end{array}$

The element $\delta$ is $\Delta^{+}$or $\Delta^{-}$depending on whether $\operatorname{det}\left(\rho^{*}(\theta)\right)$ is -1 or +1 .

REMARK. As in the remark above, we claim that we have reduced the case of Steinberg groups of type $D_{4}$ to that of Chevalley groups of type $D_{4}$. The verification is straightforward and we omit it.

2.4. Problem 2. Let $G_{1}$ be a connected group of type $A_{n}, D_{n}$, or $E_{6}$ defined over $K$ (we do not assume that $G_{1}$ is a Steinberg group) and let $G$ be the corresponding Chevalley group. We want to prove a theorem like Theorem I.2 under the assumption that $G$ and $G_{1}$ are isomorphic only (i.e. we do not require that the isomorphism be of inner type). The important fact here is that if $\rho^{*}$ exists, then $\rho^{*}: G^{*} \rightarrow O(V, B)$. 
Let $f: G_{1} \rightarrow G$ be the isomorphism. Then for $\sigma \in \Gamma, f^{\sigma} \circ f^{-1}=\theta_{\sigma} \circ I_{g_{\sigma}}$ for some $g_{\sigma} \in G$. If $(V, \rho, B)$ is an orthogonal representation of $G$ defined over $K$, then $(\rho \circ f)^{\sigma}=A_{\sigma}(\rho \circ f) A_{\sigma}^{-1}$ where $A_{\sigma}=\rho\left(g_{\sigma}\right) \rho^{*}\left(\theta_{\sigma}\right)$. Since $A_{\sigma} \in O(V, B)$, we may prove Lemma I.3 again. In the proof of Theorem I.2, the only change is in $\operatorname{det}\left(A_{\sigma}\right)$ $=\operatorname{det}\left(\rho^{*}\left(\theta_{\sigma}\right)\right)$ which may be -1 .

THEOREM II.2. Let $G_{1}$ be a connected algebraic group of type $A_{n}$, $D_{n}$, or $E_{6}$ defined over $K$ (char $K=0)$, let $G$ be the corresponding Chevalley group defined over $K$, and let $f: G_{1} \rightarrow G$ be an isomorphism between $G_{1}$ and $G$ such that $f^{\sigma} \circ f^{-1}=\theta_{\sigma} \circ I_{g_{\sigma}}$ for all $\sigma \in \Gamma$. Assume that $(V, \rho, B)$ is an orthogonal representation of $G$ defined over $K$ and assume that $\rho^{*}: G^{*} \rightarrow O(V, B)$ exists and is defined over $K$. Let $\left(V_{1} / K \#, \rho_{1}, F\right)$ be a skew-hermitian representation of $G_{1}$ defined over $K$ where $K \#=(\beta, \gamma)$ is a quaternion division algebra over $K$. Set $\operatorname{Gal}\left(K\left(\beta^{1 / 2}\right) / K\right)=\{1, \sigma\}$. Assume also that there is an absolutely irreducible representation $\theta_{1}$ : End $\left(V_{1} / K \#\right) \rightarrow$ End $(V)$ defined over $\bar{K}$ such that $\theta_{1}\left(\rho_{1}(g)\right)=(\rho \circ f)(g)$ for all $g \in G_{1}$. Then the forms $F$ and $B$ are related as follows:

(i) $\operatorname{dim} V_{1}=1 / 2 \operatorname{dim} V$.

(ii) $\delta(F)=\Delta(B)$ if $\operatorname{det}\left(\rho^{*}\left(\theta_{\sigma}\right)\right)=1$ and $\delta(F)=\beta \Delta(B)$ if $\operatorname{det}\left(\rho^{*}\left(\theta_{\sigma}\right)\right)=-1$.

\section{REFERENCES}

1. H. Boerner, Representations of groups, North-Holland, Amsterdam, 1963.

2. N. Jacobson, Lie algebras, Interscience Tracts in Pure and Appl. Math., No. 10, Interscience, New York, 1962.

3. A. I. Mal'cev, On semi-simple subgroups of Lie groups, Amer. Math. Soc. Transl. (1) 9 (1962), 172-213.

4. O. T. O'Meara, Introduction to quadratic forms, Academic Press, New York, 1963.

5. T. Ono, Algebraic groups and discontinuous groups, Nagoya Math. J. 27 (1966), 279-322.

6. I. Satake, On a certain invariant of the groups of type $E_{6}$ and $E_{7}$, J. Math. Soc. Japan 20 (1968), 322-335.

7. - Symplectic representations of algebraic groups satisfying a certain analyticity condition, Acta Math. 117 (1967), 215-279.

8. T. Tsukamoto, On the local theory of quaternionic anti-hermitian form, J. Math. Soc. Japan 13 (1961), 387-400.

University of Chicago,

Chicago, Illinois

DePaul University,

Chicago, IllinoIS 